\title{
First-order nematic-smectic phase transition for hard spherocylinders in the limit of infinite aspect ratio
}

\author{
James M. Polson and Daan Frenkel \\ FOM Institute for Atomic and Molecular Physics, Kruislaan 407, 1098 SJ Amsterdam, The Netherlands
}

(Received 11 September 1997)

\begin{abstract}
We report Monte Carlo simulations of the nematic-smectic phase transition for a system of hard spherocylinders with infinite length-to-diameter ratio. A finite-size scaling analysis suggests that this system undergoes a first-order phase transition. When combined with other simulations of the phase behavior of spherocylinders, these results suggest that the nematic-smectic phase transition is first-order for all aspect ratios. This appears to rule out the possibility of a tricritical point predicted by several density-functional theories. [S1063-651X(97)50212-1]
\end{abstract}

PACS number(s): 61.30.Cz, 64.70.Md

Some 50 years ago, Onsager showed that the nematic mesophase observed in aqueous solutions of the tobacco mosaic virus could be explained qualitatively as arising from excluded volume interactions between elongated and effectively hard particles [1]. Modeling the colloidal system as a collection of hard spherocylinders, he demonstrated that an orientationally ordered nematic phase was thermodynamically stable in the limit of infinite length-to-diameter. More recently, computer simulation studies of freely rotating spherocylinders of finite elongation have shown that these hard-core systems exhibit a remarkably rich phase behavior, with a smectic as well as a nematic liquid-crystal mesophase, in addition to two stable crystalline solid phases, a rotator solid phase, and the isotropic liquid phase [2-6]. The two most recent simulation studies of hard spherocylinders are those of McGrother, Williamson, and Jackson [5], who examined in considerable detail the phase behavior of systems with a length-to-diameter ratio of $L / D \leqslant 5$, and of Bolhuis and Frenkel [6], who mapped out the entire phase diagram in the range of $L / D=0$ to $L / D=\infty$. However, one important issue has thusfar not been resolved: the nature of the nematic-smectic $(N-\mathrm{Sm})$ transition in the limit $L / D=\infty$. For relatively short spherocylinders, the transition is clearly first order $[5,6]$. In contrast, for large $L / D$, the transition appeared to be continuous, but the results were highly sensitive to the nature of the boundary conditions employed [6].

The observation of liquid-crystalline mesophases in computer simulation studies of hard core systems inspired several theoretical studies which sought to explain the $N-\mathrm{Sm}$ phase transition in terms of excluded volume effects alone [7-10]. This was a significant development because, prior to that time, it had been assumed that smectic phases could not arise without the presence of attractive forces or flexible chains (with the notable exception of the theoretical study by Hosino et al. [11]). The density-functional theory due to Somoza and Tarazona [7] predicted a first-order $N$-Sm phase transition up to a tricritical point at $L / D=50$, after which the transition becomes continuous. Another density-functional study by Poniewierski et al. [8,9] also predicted the existence of a tricritical point for the transition, though its location in the phase diagram at $L / D=5.9$ is in quantitative disagreement with the most recent simulations [6]. In contrast, in his later bifurcation analysis study, Poniewierski [10] showed that the $N$-Sm transition in the $L / D=\infty$ limit may actually be first-order, though it was emphasized that this prediction may be an artifact due to the truncation of the virial expansion at the third order. This latter study also gave a prediction for the $N$-Sm transition density of $\rho^{*}=0.46$, in close agreement with the value of $\rho^{*}=0.47$ estimated in the simulation study of Bolhuis and Frenkel. In contrast, Somoza and Tarazona predicted $\rho^{*}=0.43$ in this limit.

In this paper, we investigate the nature of the $N$-Sm transition for spherocylinders in the $L / D=\infty$ limit. Using constant-pressure (NPT) Monte Carlo simulations, we measure the Gibbs free energy of the system as a function of a smectic order parameter $\Lambda_{s m}$. We note that a free energy barrier separating the two phases in coexistence which grows with increasing system size corresponds to the free energy of the interface between the phases, and thus, is a signature of a first-order phase transition. Thus, we seek the finite-size scaling behavior of an accurately measured free-energy profile to determine the order of the transition. We define the smectic order parameter as the magnitude of the Fourier component of the normalized density along the director,

$$
\Lambda_{s m}=\frac{1}{N}\left|\sum_{j=1}^{N} e^{i k_{z} z_{j}}\right|,
$$

where $N$ is the number of spherocylinders in the system, $k_{z}=2 \pi / \lambda_{s m}$ where $\lambda_{s m}$ is the periodicity of the smectic layers, and $z_{j}$ is the $z$ coordinate of the center of mass of the $j$ th spherocylinder, where the $z$ axis lies along the director, and thus, normal to the smectic layers. Clearly, $\Lambda_{s m}$ vanishes in the case of a spatially uniform nematic phase, and assumes a finite value less than unity for a smectic phase with density oscillations. The Gibbs free energy $G\left(\Lambda_{s m}\right)$ (to within an additive constant which is independent of $\Lambda_{s m}$ ) is given by

$$
G\left(\Lambda_{s m}\right)=-k_{B} T \ln P\left(\Lambda_{s m}\right),
$$

where $P\left(\Lambda_{s m}\right)$ is the probability that the system assumes a particular value of $\Lambda_{s m}$ at a given pressure. A practical difficulty associated with this measurement is the long time required for the system to "diffuse" through the full range of $\Lambda_{s m}$ and thus to accumulate sufficiently reliable statistics 
for the probability histogram. Consequently, we employ umbrella sampling [12], to improve the statistical accuracy of the measurement. The essence of the technique is to bias the sampling of configurational space such that configurations with a high free energy will be sampled more frequently. Furthermore, by performing a sequence of simulations in which $\Lambda_{s m}$ is biased to lie within adjacent or overlapping windows, it is possible to combine the data to form a much more accurate free-energy profile than could be obtained in the same time by direct sampling of $\Lambda_{s m}$. In the present study, we follow the approach of ten Wolde et al. [13], whereby the biasing potential for each simulation has a harmonic form $W\left(\Lambda_{s m}\right)=\frac{1}{2} k_{s m}\left(\Lambda_{s m}-\Lambda_{0}\right)^{2}$, where $k_{s m}$ determines the effective width of the window, and $\Lambda_{0}$ its position. Simulations are performed for several values of $\Lambda_{0}$ in such a way that, for a suitably chosen $k_{s m}$, the full range of $\Lambda_{s m}$ is accurately sampled. After correcting the probability measurement of each data set for the effects of the biasing, the free-energy profile can be constructed by fitting the composite data set with a polynomial.

As the freely rotating spherocylinders of the system have an infinite $L / D$ ratio, they must be appropriately scaled to an effectively finite $L / D$ in order to perform the simulation. We employ the method in the study of Bolhuis and Frenkel [6]. Briefly, we exploit the fact that the particles are almost completely aligned - the average angle the particles make with the director scales as $1 / L$. Scaling the system along the director by a factor of $L$ yields a collection of shifted cylinders (the hemispherical caps are scaled out in this compression) of constant unit length along the director, with a circular cross-section perpendicular to the director, where the shift of the cylinder is of the order of the diameter of the spherocylinder. The reduced density, $\rho^{*}=\rho / \rho_{C P}$, where $\rho_{C P}$ is the close-packing density, and the reduced pressure, $P^{*}=P /\left(\rho k_{B} T\right)$, are unaffected by the scaling. Thus, we perform Monte Carlo simulations with this scaled system in the normal NPT ensemble. Further details of the method can be found in Ref. [6].

The Monte Carlo simulations were performed at constant pressure in which volume fluctuations included independent fluctuations of the system box length along the three perpendicular coordinate axes. The simulations were performed for system sizes of $N=280,540$, and 928 spherocylinders, each with five, six, and eight layers respectively for the smectic phase. In each case, the $P\left(\Lambda_{s m}\right)$ were measured for $15-20$ values of $\Lambda_{0}$ in the range $[0,1]$ and with a harmonic width of $k_{s m} / k_{B} T=1500-3600$. These parameter values ensured a statistically relevant sampling over the full range of $\Lambda_{s m}$ with partially overlapping probability distributions for the harmonic-bias windows. Each full free-energy curve was constructed by fitting the pieces to a polynomial; the intercepts of the polynomial were independent for each segment while the higher order coefficients were the same for the full data set. In this way, the fit shifts the individual pieces to connect properly. The choice of the order of the fitting polynomial affected only very slightly the shape of the resulting curve.

Figure 1 shows the reconstructed free energy profiles and the fitted polynomials for the three system sizes at pressures of $P^{*}=5.6$ for $N=280$ and 540 , and $P^{*}=5.7$ for the $N=928$

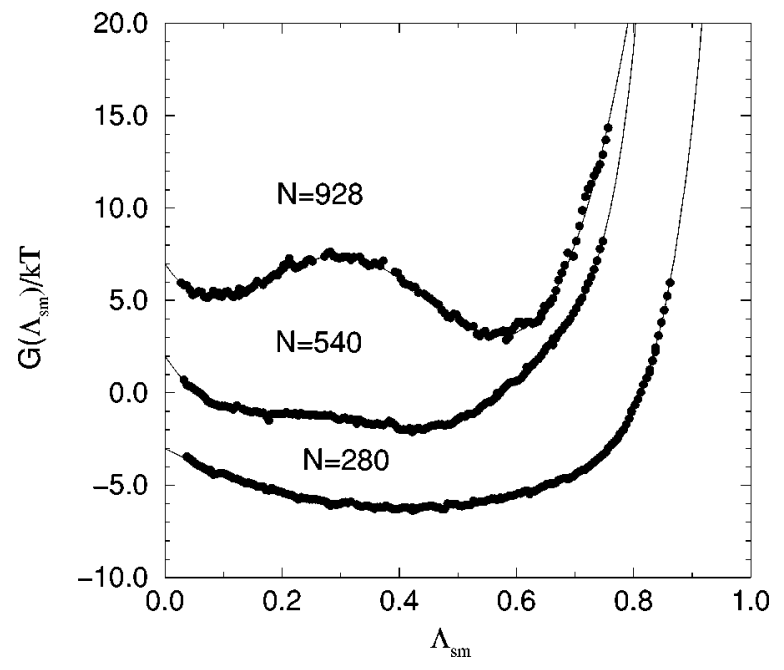

FIG. 1. Free energy profiles for hard spherocylinders of $L / D=\infty$ for three system sizes at the following pressures: $N=280, P^{*}=5.6$; $N=540, P^{*}=5.6 ; N=928, P^{*}=5.7$.

system. For the smallest system, the free energy has a flattened, slightly concave-upwards shape. The profile for $N=540$ is also fairly flat, but possibly shows a very small free-energy barrier. However, for the largest system, a freeenergy barrier of significant magnitude has developed. In this case, a smectic phase with $\Lambda_{s m} \approx 0.6$ is evident. The fact that the free energy minimum corresponding to the nematic phase is positioned slightly above $\Lambda_{s m}=0$ is an artifact of the finite size of the system. Note that the pressures were chosen to be as close as possible to the coexistence value for each system size. For the $N=928$ system, where a free energy barrier is clearly present, this is the value for which the free energy minima are equal. For the smaller systems, we define this to mean simply a value for which the profile is flat and relatively symmetric. In all cases, the shapes of the profile were found to be highly sensitive to value of the pressure: small increases (decreases) in $P$ were found to shift the global minimum to a higher (lower) value of $\Lambda_{s m}$. Predictably, this sensitivity increased with increasing system size. We note that $N=928$ system is very close to, though not precisely at, coexistence (the situation for the smaller systems is less clear-cut). Nevertheless, the qualitative picture is clear: $a$ free energy barrier develops as the system size increases, indicating that the transition is first-order. In Fig. 2 we correlate the reduced density of the system $\rho^{*}$ with the average $\Lambda_{s m}$ for each of the sampling windows for each of the three systems studied here. In each case there is monotonic increase in $\Lambda_{s m}$ with $\rho^{*}$. Note that the upwards shift of the largest system data is simply a result of the slightly higher pressure used in this case. From this graph, we see that for the $N=928$ system there is approximately a $2 \%$ density difference corresponding to the free energy minimum values of $\Lambda_{s m}$, i.e., a $2 \%$ discontinuity between the density of the nematic and smectic phases. Figure 3 shows a snapshot of the system in a simulation carried out for a value of $\Lambda_{0}$ fixed near the top of the free energy barrier, and further corroborates our conclusion of a first-order $N$-Sm phase transition. The snapshot shows the coexistence of nematic and smectic domains in the system. The free energy of the interface between these domains corresponds to the the height of the free energy barrier. 


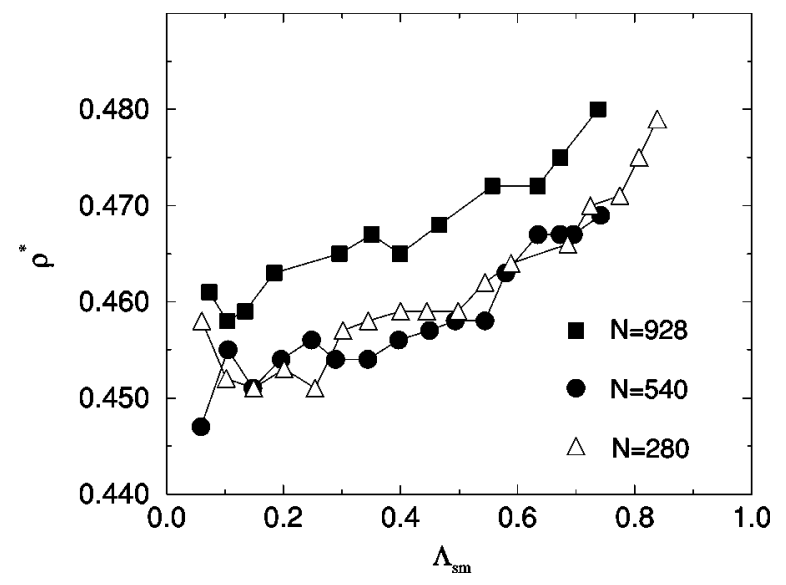

FIG. 2. Density vs smectic order parameter corresponding to the three systems in Fig. 1. Each point represents a separate simulation for a window with a position determined by $\Lambda_{0}$, and a width determined by $k_{s m}$. Both $\Lambda_{s m}$ and $\rho^{*}$ are average values for each sampling window.

In summary, our simulation results strongly suggest that the $N$-Sm phase transition for a system of hard spherocylinders in the $L / D=\infty$ limit is first order. Note that while it would be desirable to carry out simulations for larger system sizes to check that the free energy barrier scales as $N^{2 / 3}$ as the thermodynamic limit is approached, this is currently not possible: attempts to perform simulations with larger system sizes were found to be prohibitively time consuming.

It is instructive to compare the results of this study with those of Bolhuis and Frenkel [6]. In the latter study, the presence of hysteresis in the nematic and smectic branches of the equation of state of spherocylinders in the limit of infinite

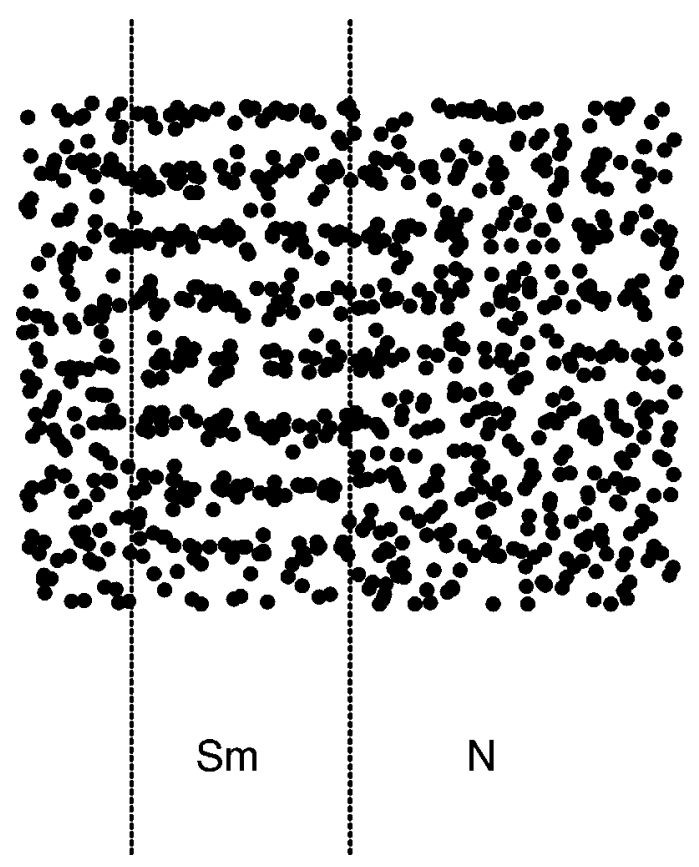

FIG. 3. Snapshot of the projection of the centers of mass of a system of $N=928$ spherocylinders with the nematic director along the vertical axis. The snapshot is from a simulation with a biasing potential parameter of $\Lambda_{0}=0.35$, a value corresponding to the top of the free energy barrier in Fig. 1. Coexisting smectic-A (Sm) and nematic $(\mathrm{N})$ domains are labeled. aspect ratio was found to depend on the type of boundary conditions employed. In the case of regular periodic boundary conditions, some hysteresis was observed, while in the case of shifted periodic boundary conditions, in which all layers in the smectic phase are essentially connected, no hysteresis was observed. Note that the system size in that study was $N=540$. We repeated the NPT-MC simulations for this system, and found that the apparent hysteresis disappeared if the length of the simulations, in particular, the number of volume changing moves, was increased by a factor of ten. The lack of hysteresis is clearly consistent with the negligible free energy barrier shown in Fig. 1. However, this feature is itself a result of the small system size. It is expected that the hysteresis would be clearly manifest for a larger system with a significant barrier, and with metastable states covering a sufficiently wide range of pressures.

Finally, we note that the $N$-Sm transition for hard spherocylinders was shown previously to be first order for finite $L / D$ values, and, from the present study, is shown to be very likely also first order in the $L / D=\infty$ limit. These limiting cases, therefore, rule out the existence of a single tricritical point (though they are not inconsistent with the more unlikely presence of two tricritical points). Thus, the $N$-Sm phase transition very probably is first order over the entire $L / D$ range that it covers.

As a complement to our investigation of the nature of the $N$-Sm transition of infinitely long rigid spherocylinders, we discuss briefly preliminary simulation results describing the role that flexibility plays for this system. Recently, there has been growing interest in the effects of flexibility on the $N$-Sm phase transition of hard-core systems. An important recent experimental study of suspensions of fd virus particles, which can be viewed approximately as semiflexible hard rods, investigated the nature of cholesteric-smectic transition [14]. (The chirality of the cholesteric phase is unimportant, and, thus, the transition is essentially $N$-Sm in nature). It was argued that the presence of flexibility acts to increase the volume fraction at the transition, decrease the smectic periodicity relative to the particle length, and to drive the transition first order. This work prompted two theoretical studies: one in which the particles are highly flexible ( $l^{*} / L \approx \lambda / L \ll 1$, where $\lambda$ is the deflection length, and $l^{*}$ is a similarly defined "screening length") [15], and one for slightly flexible particles $(\lambda / L>1)$ [16]. Both theories were able to reproduce the basic experimental results. As well, a recent molecular dynamics simulation study of semi-flexible hard rods composed of spherocylinder segments also found evidence for a $N$-Sm transition shifted to higher volume fractions [17].

We performed NPT-MC simulations for a system of $N=320$ semiflexible rods composed of six segments, where each segment was a $L / D=\infty$ rod scaled in the manner described above. Collective moves involving the simultaneous tilting of all particles in smectic layers, as well as collective displacements of segment joints at nearby positions along the director axis, were introduced to increase the equilibration rate and decrease the timescale of fluctuations. Despite these attempts, the system evolved very sluggishly, and equilibration runs of $\sim 10^{5} \mathrm{MC}$ cycles were required. Initial configurations in both the nematic and smectic phases were employed. For a flexibility given by $\lambda / L=0.46$, we observe a 
stable nematic phase with a density of $\rho^{*}=0.51$ at $P^{*}=10.0$, and a stable smectic phase at a density of $\rho^{*}=0.55$ at $P^{*}=12.0$; thus, a $N$-Sm phase transition lies within this density range. From comparable simulations performed on smaller systems, we observe that the transition density increases with increasing system size, i.e. the effect of a relatively small system size is to stabilize the more ordered (smectic) phase. Consequently, the observed transition density for the $N=320$ system of $\rho^{*} \in[0.51,0.55]$ is a lower limit for the value anticipated for larger system sizes. This compares with the observed $N$-Sm coexistence density range for rigid rods of $\rho^{*} \in[0.46,0.47]$ for $N=928$. Thus, the ef- fect of flexibility on a system of hard rods with $L / D=\infty$ is to shift the density at the transition to higher values, consistent with experimental, theoretical and computer simulation results for finite- $L / D$ particles.

This work is part of the research program of the "Stichting Fundamenteel Onderzoek der Materie", (FOM) and is supported by "Nederlandse Organisatie voor Wetenschappelijk Onderzoek" (NWO). J.P. acknowledges the financial support provided by the Computational Materials Science program of NWO. We would like to thank Richard Sear and Blandine Jérôme for a critical reading of the manuscript.
[1] L. Onsager, Ann. (N.Y.) Acad. Sci. 51, 627 (1949).

[2] D. Frenkel, J. Phys. Chem. 92, 3280 (1988).

[3] D. Frenkel, H. N. W. Lekkerkerker, and A. Stroobants, Nature (London) 332, 882 (1988).

[4] J. A. C. Veerman and D. Frenkel, Phys. Rev. A 41, 3237 (1990).

[5] S. C. McGrother, D. C. Williamson, and G. Jackson, J. Chem. Phys. 104, 6755 (1996).

[6] P. Bolhuis and D. Frenkel, J. Chem. Phys. 106, 666 (1997).

[7] A. M. Somoza and P. Tarazona, Phys. Rev. A 41, 965 (1990).

[8] A. Poniewierski and R. Holyst, Phys. Rev. A 41, 6871 (1990).

[9] A. Poniewierski and T. J. Sluckin, Phys. Rev. A 43, 6837 (1991).
[10] A. Poniewierski, Phys. Rev. A 45, 5605 (1992).

[11] M. Hosino, H. Nakano, and H. Kimura, J. Phys. Soc. Jpn. 46, 1709 (1979).

[12] G. M. Torrie and J. P. Valleau, Chem. Phys. Lett. 28, 578 (1974).

[13] P. R. ten Wolde, M. J. Ruiz-Montero, and D. Frenkel, J. Chem. Phys. 104, 9932 (1996).

[14] Z. Dogic and S. Fraden, Phys. Rev. Lett. 78, 2417 (1997).

[15] A. V. Tkachenko, Phys. Rev. Lett. 77, 4218 (1996).

[16] P. van der Schoot, J. Phys. (France) II 6, 1557 (1996).

[17] P. Bladon and D. Frenkel, J. Phys.: Condens. Matter 8, 9445 (1996). 